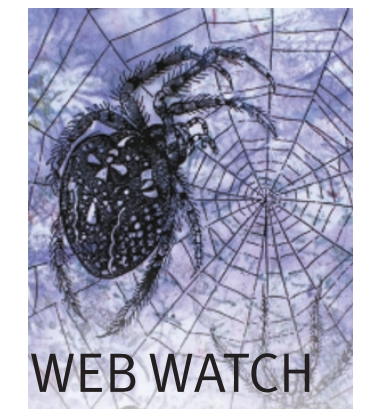

- http://wuw.biocompare.com

\section{Buyer's guide}

Do you ever want to buy an enzyme or kit but end up being bewildered by which one to choose from the

increasingly large range on offer? Well, Biocompare -

a company based in

California, United States aims to help by being "The buyer's guide for life scientists". It was started a

few years ago by life scientists who wanted

better information in order to compare reagent

purchasing, and on this web site you can browse

products that relate to cell

biology, molecular biology and tissue culture, to name just a few. The company's mission is "to facilitate scientific discovery by providing objective product information to the life science community".

And this site is not just

about browsing products -

it has evolved to include

several other useful and

freely accessible sections,

including New

Technologies, News,

Professional Reviews and

Technology Spotlights. You

can view News as either

'Top Stories', or perhaps more conveniently under

categories relating to your research interests. The

Professional Reviews

contain a synopsis, which succinctly summarizes the review information under the headings 'Product', 'The

Good', 'The Bad' and 'The

Bottom Line'.

The web site is easy to use and navigate, and is consistently and frequently updated. There is a simple search facility on the home page, and the search results are listed under the sections mentioned above. By stopping scientists having to search through print catalogues to find the product they need, Biocompare aims to give them more time to focus on their valuable work.

Rachel Smallridge

\title{
RNA PROCESSING
}

\section{Nonstop destruction}

How do you deal with messenger RNAs that just don't know when to quit? Cells can create transcripts that contain no stop codons for the translation machinery to recognize, which can lead to the synthesis of abnormal and potentially harmful proteins. But according to two reports in Science, cells recognize these so-called 'nonstop' mRNAs and can destroy them using a mechanism called nonstop decay.

The cell has evolved a remarkable array of quality-control mechanisms to ensure gene expression occurs correctly, such as nonsense-mediated mRNA decay (NMD), which detects mRNAs that contain premature termination codons and stops the formation of truncated proteins.

But what happens in the opposite case, when no stop codon exists? Frischmeyer and colleagues constructed a nonstop-PGK1 construct in which all in-frame termination codons were removed - and found that these 'nonstop' transcripts were just as unstable in Saccharomyces cerevisiae cells as a nonsense form of the gene.

However, the nonstop transcripts were still unstable in yeast mutants that lacked factors required for both the NMD pathway and for the

\section{CELL POLARITY}

\section{Building site}

Multi-domain scaffolding proteins are important for cell polarity because they target proteins to precise subdomains. Margolis's group has now identified a new multiprotein complex that localizes to tight junctions and is therefore a strong candidate for regulating epithelial cell polarity.

The authors found that Pals1, a membrane-associated guanylate kinase (Maguk) scaffolding protein with several protein-interaction domains, localizes to tight destruction of normal mRNAs, yet required translation of the mRNA for the decay to occur (as shown by cycloheximide treatment, which inhibits protein synthesis, or by depletion of charged transfer RNAs) - so the destruction of nonstop transcripts had to be occurring through a different pathway.

The most obvious candidate is the exosome, a collection of proteins with a $3^{\prime}-5^{\prime}$ exoribonuclease activity that processes RNAs, such as ribosomal RNA, small nuclear RNA and small nucleolar RNA. Van Hoof et al. showed that a yeast mutant for Ski4, one of the core exosome subunits that specifically disrupts cytoplasmic $3^{\prime}-5^{\prime}$ degredation of mRNA, stabilized the nonstop-PGK1 at least sixfold. The nonstop transcripts were also stabilized by mutant forms of Ski7, which is related to the translation elongation factor EF1A and the translation temination factor eRF3, and is one of two other factors that are also required for exosome-mediated degradation. Transcriptional pulse-chase studies using transcripts with differing lengths of poly(A) tails suggested that the mechanism used to degrade

junctions, and then identified one potential protein - Pals1associated tight junction protein (PATJ) — that might target it there. PATJ binds to a distinct domain (L27N) of Pals1 through a new protein-protein interaction domain that the authors called a Maguk recruitment (MRE) domain.

PATJ was shown to be part of a ternary complex that binds not only Pals1, but also CRB1, the human homologue of Drosophila melanogaster Crumbs. But PATJ and CRB1 don't bind directly to each other, which indicates that Pals1 probably functions as an adaptor. Similarly, in Drosophila, Crumbs binds the Pals1 orthologue, Stardust, but doesn't

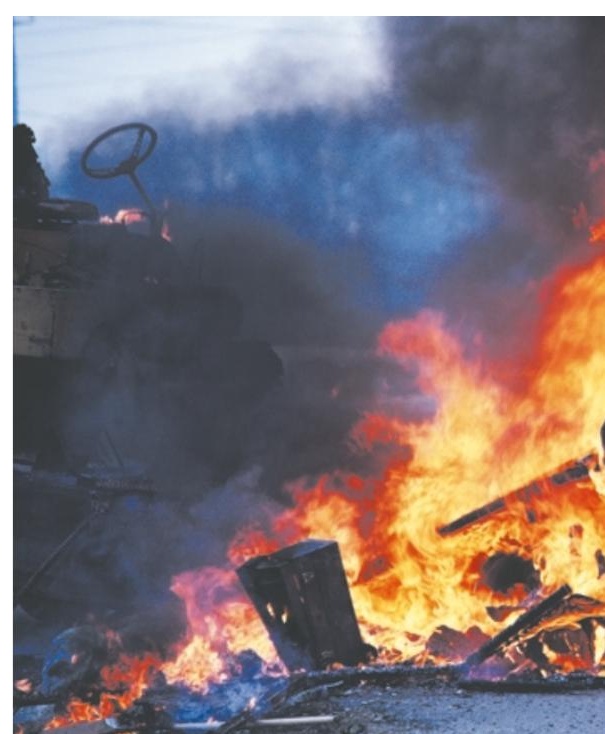

the nonstop mRNAs starts at the $3^{\prime}$ end of the poly(A) tail.

Together, these data provide a model for how the cell detects and destroys nonstop mRNAs. When the ribosome reaches the end of the nonstop mRNA strand, the exosome homes in on the stalled ribosome. Ski7 associates with the cytoplasmic form of the exosome, which then degrades the mRNA, starting from the $3^{\prime}$ end of the poly(A) tail. Given the similarity of Ski7 to EF1A and eRF3 - which interact with the A site of ribosomes that contain a sense or nonsense codon, respectively Ski7 might distinguish nonstop from normal mRNAs by binding to the empty A site of ribosomes that have reached the $3^{\prime}$ end of mRNAs.

Many questions remain but the authors say that nonstop decay might be a valuable and necesssary mechanism. It could be required for mRNAs containing a $3^{\prime}$-end

bind the PATJ homologue, Discs Lost.

Discs Lost and Crumbs are both essential for cell polarity and have also been implicated in the localization of adherens junctions. So, although the exact function of the Pals1 complex needs further clarification, its conservation from fly to human indicates the importance of this complex.

\section{Katrin Bussell}

(2) References and links ORIGINAL RESEARCH PAPER Roh, M. H. et al. The Maguk protein, Pals1, functions as an adaptor, linking mammalian homologues of Crumbs and Discs Lost. J. Cell Biol. 157 161-172 (2002)

FURTHER READING Rashbass, P. \& Skaer, H. Cell polarity: Nailing Crumbs to the scaffold. Curr. Biol. 10, 234-236 (2000)

WEB SITE

Ben Margolis' laboratory:

http://www.med.umich.edu/cmb/margolis.htm 


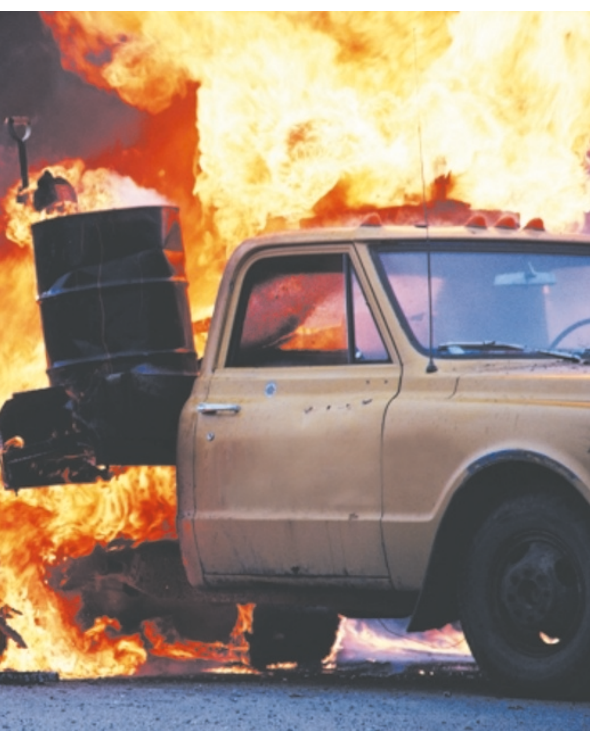

processing signal located upstream of the termination codon (as is found in $1.2 \%$ of expressed sequence tags from S. cerevisiae), or the nonstop mRNAs might be created when $3^{\prime}$-end formation takes place upstream of the normal termination after RNA polymerase pausing, when ribosomes pause at rare codons or normal termination codons, or $3^{\prime}-5^{\prime}$ decay is initiated on ribosome-bound mRNA. Nonstop decay is also initiated after manipulations that increase readthrough of termination codons, as occurs after administration of aminoglycoside antibiotics.

Simon Frantz

20) References and links ORIGINAL RESEARCH PAPERS Frischmeyer, $P$. A. et al. An mRNA surveillance mechanism that eliminates transcripts lacking termination codons. Science 295, 2258-2261 (2002) | van Hoof, A., Frischmeyer, P. A., Dietz, H. C. \& Parker, R. Exosome-mediated recognition and degradation of mRNAs lacking a termination codon. Science 295, 2262-2264 (2002)

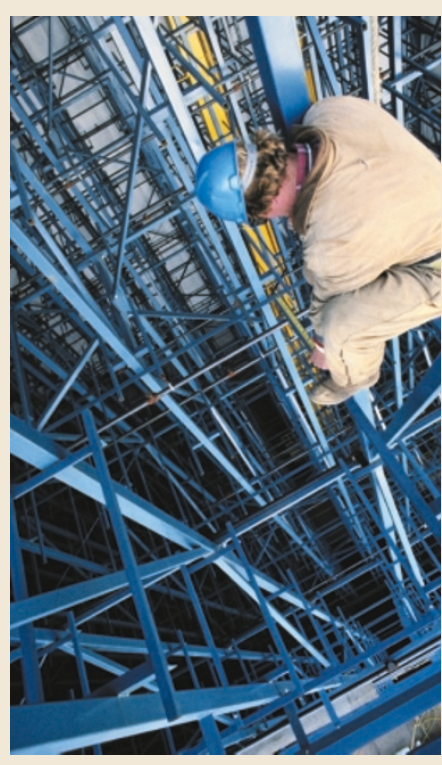

H I G H L I G H T S

CELL CYCLE

\section{Daughter control}

Controlling cytokinesis so that two identical daughter cells are formed from mitosis is one of the many crucial steps in the cellcycle pathway. And despite intense study, the mechanisms involved in this process are still unknown. But enter a new player, report Fujikawa and colleagues in Proceedings of the National Academy of Sciences. They have found that Vav3 is regulated in a cell-cycledependent manner and could be involved in cytokinesis regulation.

The Vav family are guanine nucleotide exchange factors for Rho-family GTPases. Although the three members of the Vav family (Vav1-3) are highly similar (50-70\% homology at the amino-acid level) and share certain domains, recent studies had shown that Vav3 might regulate RhoGTPases in a different manner to Vav1 and Vav2.

The exact function of Vav3 is unknown, but in vitro studies had shown that Vav3 was a specific activator for RhoA — which is known to be involved in cytokinesis - so Fujikawa and colleagues wanted to see if Vav3 also had a role in this process. Analysis of the expression and intracellular distribution of Vav3 in HeLa cells showed that levels of the protein rapidly increased during mitosis - Vav3 could be detected after the disappearance of the nuclear membrane (during the transition from prophase to prometaphase) and then slowly decreased throughout anaphase towards the onset of cytokinesis. RNase protection assays confirmed this expression pattern and showed it differed from that of its family members Vav3 messenger RNA levels were upregulated during mitosis, whereas levels of Vav2 mRNA did not change (Vav1 was not studied as it is not expressed in HeLa cells).

The importance of this regulation became apparent as the enforced expression (by transfection of full-length cDNA constructs in HeLa cells) of Vav3, but not Vav1 or Vav2, disrupted cell division and led to the production of multinucleated cells, which indicates a block in cytokinesis. This effect seems likely to involve the activation of endogenous RhoA, as production of these multinucleated cells could be stopped by coexpression of Vav3 with a dominant-negative mutant of RhoA, but coexpression with mutants of other Rho-family members, such as Racl or Cdc42, had no effect.

Further characterization of Vav3 showed both differences and similarities to the other Vav proteins. The authors were surprised to find that deletions of the amino-terminal region of Vav3 did not affect the ability to produce multinucleated cells, as similar deletions in Vav1 and Vav2 had affected their activity. However, like Vav1, the activity of Vav3 is regulated by phosphorylation of a conserved tyrosine residue at position 173 (174 in Vav1), as a Y173F substitution completely abolished the ability to induce multinucleated cells.

So, the authors conclude that Vav3 could be an upstream regulator of RhoA during cytokinesis. This not only provides a new target for studies in cytokinesis control but, given the cell-cycledependent expression pattern of Vav3, also identifies a new mode of regulation among the Vav proteins.

Simon Frantz

\section{(2) References and links}

ORIGINAL RESEARCH PAPER Fujikawa, K. et al. Vav3 is regulated during the cell cycle and effects cell division. Proc. Natl Acad. Sci. USA 99, 4313-4318 (2002)

FURTHER READING Bustelo, X. R. Vav proteins, adaptors and cell signaling. Oncogene $\mathbf{2 0}$ 6372-6381 (2001)
CELL SIGNALLING

\section{The}

MIDAS touch

Although integrins bind to a structurally diverse range of ligands, most of these ligands contain the sequence Arg-Gly-Asp (RGD). The structural basis for this cation-dependent interaction has been unclear, but now, in Science, Arnaout and colleagues report the crystal structure of the extracellular segment of integrin $\alpha \mathrm{V} \beta 3$ in complex with an RGD ligand.

Integrins are composed of an $\alpha$ and $\beta$ subunit - both type I membrane proteins with large extracellular domains - and fall into two classes depending on the presence or absence of an $\alpha A$ domain. Studies on the structure of $\alpha A$ have previously shown that a metal-ion-dependent adhesion site (MIDAS) at the ligand-binding interface is required for ligand interactions with $\alpha \mathrm{A}$-containing integrins. In $\alpha \mathrm{V} \beta 3$, which lacks $\alpha \mathrm{A}$, Arnaout and co-workers found that ligand binding is mediated by an $\alpha$ A-like $\beta$ A domain in $\beta 3$. Surprisingly, $\beta A$ acquires two cations on complex formation - one in MIDAS and another in a ligandassociated metal-binding site (LIMBS). They propose that LIMBS, which does not directly contact RGD, stabilizes the ligand-binding surface.

The authors found that RGD binds at the major interface between $\alpha \mathrm{V}$ and $\beta 3$, and induces both tertiary and quaternary structural changes, which probably represent "a minimalist view" of the changes in integrins that are required for cell signalling. Although the ligand used here was synthetic, its RGD motif is almost conformationally identical to that present in a known natural ligand, which indicates that this structure can be used to understand integrin interactions with other RGD-containing ligands.

Rachel Smallridge

\section{(2) References and links} ORIGINAL RESEARCH PAPER Xiong, J.-P. et al. Crystal structure of the extracellular segment of integrin $\alpha \vee \beta 3$ in complex with an Arg-Gly-Asp ligand. Science 296, 151-155 (2002) 